\title{
RIESGO DE ESTRÉS EN ESTUDIANTES DE ENFERMERÍA DURANTE LAS PRÁCTICAS CLÍNICAS
}

RISK OF STRESS IN NURSING STUDENTS IN CLINICAL PRACTICE

Collados Sánchez, Juana María.

E-mail: juanamar.collados@hotmail.com

García Cutillas, Nuria.

E-mail: nugarcu@hotmail.com

\section{RESUMEN}

El estudiante tiene que afrontar nuevas situaciones para las cuales no está suficientemente preparado. Esta falta de preparación también marca el nivel de estrés en las diferentes situaciones, es decir lleva al estudiante a vivir con altos niveles de estrés.

OBJETIVO: El objetivo del presente estudio es indagar sobre los factores que resultan fuente de estrés en las prácticas clínicas para los estudiantes de enfermería.

MÉTODO: Es un estudio realizado en la Escuela de Enfermería de la Universidad de Murcia durante el mes de Marzo del curso 2010/2011. La muestra es de 30 alumnos pertenecientes a, los cursos primero, segundo y 
tercero de la Diplomatura, a los cuales se les paso una encuesta validada mediante un previo pretest.

RESULTADO: Después de haber elaborado el trabajo, vemos que lo que más preocupa a los alumnos de primer curso es el desconocimiento ante una situación clínica, a los alumnos de segundo es la impotencia e incertidumbre ante situaciones determinadas y el riesgo a sufrir contagio, daños o lesiones en la relación con el paciente $y$, finalmente, a los alumnos de tercero lo que más estrés les provoca es la valoración de sus prácticas.

CONCLUSIONES: Las soluciones que proponemos a todos estos factores de estrés es un esfuerzo continuado por parte de los alumnos reforzando los conocimientos adquiridos en clase, reduciendo los grupos de trabajo, conseguir una metodología de trabajo y finalmente conocer el funcionamiento del lugar donde se desarrollan las prácticas clínicas.

Palabras clave: Estrés, estudiantes, prácticas.

\section{ABSTRACT}

The student has to face new situations for which they are not ready enough. This lack of training also marks the stress level in different situations, that is, this leads students to live with high stress levels.

OBJETIVE: The aim of this study is to investigate about the facts that result in stress on clinical practices for nursing students.

METHOD: This is a study made by the Nursing School of the University of Murcia during the month of March in the academia year 2010/2011. The sample has been made to 30 students that belong to the first, second and third level of the degree, to whom a survey validated by a previous test was given.

RESULT: Alter doing the job, we see that the thing that more upset first level students is ignorance in a clinical situation, for second level students is the impotente and uncertainty on particular situations and the risk of suffering from infection, damages or injuries in patience relationship. And finally, to third 
level students the more stressful thing is their practice evaluation.

CONCLUSIONS: The solutions that we propose to all those stress factors is a constant effort done by the students reinforcing all the acquire knowledge in the classroom, reducing the working groups, to get a job methodology and finally to know the way the place where the practices take place works.

Key words: Stress, Students, Practicals.

\section{INTRODUCCIÓN}

En la formación de los/las alumnos/as de enfermería, además del componente teórico del que consta la formación durante todos los cursos, gran parte de los créditos necesarios para obtener la titulación son prácticos. El objetivo de las prácticas clínicas es que se obtenga un aprendizaje progresivo, complementario y realista de la futura profesión y también aplicar los conocimientos teóricos adquiridos ${ }^{[1]}$. Como medio de interrelación entre ambos conocimientos, los alumnos realizan la parte práctica tutelados por enfermeros asistenciales en diferentes unidades hospitalarias y en atención primaria.

Es un punto bastante importante la realización de dichas prácticas en enfermería no solo por el amplio campo de actuación enfermero en todas las etapas de la vida, abarcando también la prevención y educación para la salud, sino también porque se adquiere un desarrollo de habilidades y destrezas que no se consiguen en el aula y unas actitudes necesarias para ser futuros profesionales sanitarios. En las que, los/las alumnos/as suelen salir satisfechos tras estos períodos prácticos. ${ }^{[2],[3]}$

En un estudio se ha observado que, antes de iniciar las prácticas clínicas, la mayoría de los/las alumnos/as creen que el estrés va a estar presente en el transcurso de las mismas ${ }^{[4]}$, y entre sus temores principales están el considerar que no están preparados, que pueden cometer fallos con los pacientes y el miedo a contagiarse de alguna enfermedad. ${ }^{[5]}$

Lo que ocurre con frecuencia, en el caso del estudiante, es el hecho de tener que afrontar nuevas situaciones para las cuales no está suficientemente 
preparado. Esta falta de preparación también marca el nivel de estrés en las diferentes situaciones, es decir lleva al estudiante a vivir con altos niveles de estrés. Lo que nos lleva a plantearnos la necesidad de estudiar los factores estresantes y la manera de actuar frente a ellos. Por lo que los objetivos del presente estudio nos lleva a indagar sobre los factores que resultan fuente de estrés en las prácticas clínicas para los estudiantes de enfermería, realizar un análisis diferencial de la valoración que hacen los estudiantes de enfermería, de los factores estresantes académicos y de los niveles percibidos de respuesta de estrés en función del sexo y en función del curso, y comparar nuestros resultados con otros estudios realizados por otros autores.

\section{MATERIAL Y METODOLOGÍA}

Se trata de un trabajo de investigación descriptivo y observacional transversal, cualitativo y cuantitativo, prospectivo y aleatorio, realizado con una muestra total de 30 participantes, estudiantes de la Escuela de Enfermería de la Universidad de Murcia en el curso 2010/2011, de los cuales 10 son de $1^{\circ}, 10$ son alumnos de $2^{\circ}$ y otros 10 son de $3^{\circ}$ curso. Temporalidad: Se pasó una encuesta validada mediante un previo pretest, durante el mes de Marzo de 2011.

Instrumento de medición: La encuesta definitiva consta de un total de 12 ítems que pretenden medir aspectos demográficos de la muestra y los distintas fuentes de estrés. Las respuestas a cada uno de los ítems se realizaron sobre una escala de Likert de cuatro puntos desde "Nada" (1), "Algo (2), "Bastante" (3), "Mucho" (4).

La recopilación de datos se ha llevado a cabo mediante el programa informático Excel.

\section{RESULTADOS}

Los resultados del estudio nos muestran que, lo que más preocupa a los alumnos es encontrarse ante situaciones en las que no saben como actuar, así como el riesgo de sufrir contagios y la evaluación de las prácticas clínicas. 
Uno de los factores causantes que pueden llevar a estas situaciones de estrés puede encontrarse en la falta de conocimientos.

Observamos que la edad media de la muestra es de 21 años (Tabla 1), presentándose un mayor porcentaje de mujeres (76.6\%) entre los componentes de la muestra (Tabla 2).

La media de los resultados obtenidos de los tres cursos, observamos que los ítems que más estrés les produce a los alumnos, es el desconocimiento ante una situación clínica, riesgo a sufrir contagios, daños o lesiones en relación con el paciente y el riego a dañar al paciente (Tabla 3).

Respecto a los resultados obtenidos por curso y sexo de la Diplomatura de Enfermería, contemplamos que en los alumnos de primer curso lo que menos estrés les causa son las relaciones con los familiares y el exceso sobrecarga de trabajo, produciéndoles mayor estrés el desconocimiento ante una situación clínica. En cuanto a los alumnos de segundo lo que menos estrés les produce son las relaciones con los familiares, y lo que más estrés les causa es la impotencia e incertidumbre ante situaciones determinadas y riesgo a sufrir contagios, daños o lesiones en relación con el paciente. Finalmente a los alumnos de tercero no les produce estrés el manejo de nuevas tecnologías, y sorprendentemente lo que más les produce estrés son los resultados de la evolución de las prácticas clínicas (Tabla 4).

De los datos obtenidos por sexos, el mayor estresor de los hombres es los resultados de la evaluación de las prácticas, en cambio para las mujeres es el desconocimiento ante una situación clínica y el riesgo a sufrir contagio, daños o lesiones en la relación con el paciente.

Así mismo, lo que menos estrés causa a las mujeres y hombres, es las relaciones con los familiares del paciente (tabla 5 ).

\section{DISCUSIÓN}

En la bibliografía revisada, hemos observado, que las situaciones percibidas como más estresantes fueron "Hacer mal mi trabajo y perjudicar al paciente", "Pincharme con una aguja infectada" y "Confundirme de 
medicación".Expresan una considerable percepción del estrés, siendo mayor en segundo que en primero, en las alumnas que los alumnos ${ }^{[6]}$.

En nuestros resultados observamos que coincidimos en cuanto al nivel de estrés, que es mayor en las alumnas que en los alumnos, y también en las situaciones percibidas como más estresantes, siendo el riesgo de sufrir contagios. Sin embargo el resultado obtenido en el estudio difiere de estos autores en cuanto a otras situaciones percibidas como más estresantes, como son "Desconocimiento ante una situación clínica", "Impotencia e incertidumbre ante situaciones determinadas" y "Resultados de la evaluación de las practicas".

Antolín Rodríguez ${ }^{[7]}$, destaca cómo la situación percibida en los diferentes cursos y más estresante "Hacer mal mi trabajo y perjudicar al paciente".

Las principales fuentes de estrés, tanto al principio de la carrera como al final, son en orden de importancia, la falta de competencia, impotencia e incertidumbre, dañarse en la relación con el enfermo, implicación emocional, no controlar la relación con el enfermo, contacto con el sufrimiento y relación con los compañeros ${ }^{[8]}$. A lo largo de la diplomatura hay una disminución significativa en la puntuación de las principales fuentes de estrés.

En cuanto a lo expuesto, en nuestros resultados observamos que hay una disminución significativa en el nivel de estrés a lo largo de la carrera y algunos de los factores más estresantes son "La impotencia e incertidumbre", no coincidiendo con otros autores en el resto de factores estresantes, ni en que dichos factores sean los mismos en los distintos cursos.

\section{CONCLUSIONES}

Se define el estrés como los esfuerzos cognoscitivos y conductuales, constantemente cambiantes que se desarrollan para las demandas específicas externas e internas, que son evaluadas como excedentes o desbordantes de los recursos del individuo, es decir, a los esfuerzos que realiza una persona para anticiparse, desafiar o cambiar las condiciones para alterar una situación estresante. 
Observando los resultados obtenidos de las encuestas planteamos una serie de soluciones a estos problemas. Respecto a los alumnos de primero, lo que más les produce estrés es el desconocimiento ante una situación clínica, ya que muchos de estos alumnos no han tenido contacto con el medio sanitario; por lo que deberán hacer más casos prácticos de situaciones reales, realizar exámenes de autoevaluación y tener más horas prácticas. A los alumnos de segundo lo que más estrés les causa es la impotencia e incertidumbre ante situaciones determinadas y el riesgo a sufrir contagio, daños o lesiones en la relación con el paciente; las soluciones propuestas al respecto son adquirir conocimientos básicos sobre protección, tener conocimientos sobre enfermedades contagiosas y su modo de transmisión, saber controlar la situación, cursos o asignaturas de libre configuración sobre medidas de protección y de enfermedades contagiosa y realizar las prácticas clínicas con un tutor individualizo que de seguridad en cada situación. Para concluir, a los alumnos de tercero lo que más estrés les produce son los resultados de la evaluación de sus prácticas, entendemos que es normal este estrés, ya que se encuentran en su último año de la Diplomatura, por lo que proponemos la explicación de las diferentes prácticas que van a realizar durante su último curso y asentamiento de los conceptos de las diferentes materias antes de acudir a las prácticas clínicas.

Como medida general para todos los cursos, deben de tener un esfuerzo continuado por parte de los alumnos, reforzando los conocimientos adquiridos en clase, reducir el número de alumnos en los grupos de trabajo y prácticas, para los alumnos que realizan prácticas clínicas deben tener en cuenta la colaboración en el equipo de trabajo, tener una metodología de trabajo, ya que les dará más seguridad y finalmente conocimiento sobre el funcionamiento del lugar donde se desarrollan las prácticas clínicas. 


\section{BIBLIOGRAFÍA}

1. Ferrer Pascual, Ma A. Análisis situacional de las prácticas clínicas en la escuela de enfermería de Soria. Metas, 2002; 44:18-22.

2. Zapico F. Aprendizaje y satisfacción de los estudiantes de enfermería en las prácticas clínicas. Hospital. Enfermería Clínica, 1997; 7:16-24.

3. Juanola MD. Aprendizaje y satisfacción de los estudiantes de enfermería en las prácticas clínicas. Atención Primaria de Salud. Enfermería Clínica,1998; 8:254-62.

4. Alemán S. Las prácticas de enfermería ¿crecimiento o deterioro? Situación de los alumnos previa a las prácticas. Enferm Cient. 1998.

5. Gutiérrez MI. Las prácticas clínicas de enfermería: perspectiva de los estudiantes de primer curso. Metas de Enfermería. 2002; 49:50-3.

6. López Medina IM. Percepción del estrés en estudiantes de enfermería en las prácticas clínicas. Enfermería Clínica, 2005; 15(6):307-13.

7. Rodriguez A. Situaciones de las prácticas clínicas que provocan estrés en los estudiantes de enfermería. Enfermería Global, 2007.

8. Zupiria Gorostidi X. Fuentes de estrés en la práctica clínica de los estudiantes de enfermería: evolución a lo largo de la diplomatura. Enfermería Clínica, 2006; 16(5):231-37.

\section{BIBLIOGRAFÍA COMPLEMENTARIA}

- Ticona Benavente SB. Nivel de estrés y estrategias de afrontamiento en estudiantes de la facultad de enfermaría- Unsa Arequipa. Enfermería Global, 2006.

- Sánchez M. Estrés de los estudiantes de enfermería en el período práctico. Enferm Cient. $1992 ; 127: 43-6$.

- Amat V. Estrés en estudiantes de enfermería. Rev Rol Enf. 1990; 140:75-8.

- Vila M. Visión del estudiante de enfermería sobre sus prácticas clínicas. Metas de Enfermería. 2001; 40:25-31. 


\section{ANEXOS}

Tabla 1. Media de la edad de los encuestados por curso y total.

MEDIA DE EDAD:

\begin{tabular}{|c|c|c|c|}
\hline 10 & 20 & $3^{\circ}$ & TOTAL \\
\hline 19,2 & 21,5 & 23,7 & 21,67 \\
\hline
\end{tabular}

Tabla 2. Porcentaje de sexo de los encuestados por curso y total.

\% DE SEXO DE ALUMNOS

\begin{tabular}{|l|c|c|c|c|}
\hline SEXO & 10 & 20 & 30 & TOTAL \\
\hline HOMBRE & $20 \%$ & $40 \%$ & $10 \%$ & $23,33 \%$ \\
\hline MUJER & $80 \%$ & $60 \%$ & $90 \%$ & $76,67 \%$ \\
\hline
\end{tabular}

Tabla 3. Media de los resultados obtenidos por curso y total.

\begin{tabular}{|c|c|c|c|c|}
\hline MEDIAS & 10 & 20 & 30 & TOTAL \\
\hline Manejo de nuevas tecnologías & 2,9 & 2,1 & 1,8 & 2,27 \\
\hline Desconocimiento ante una situación clínica & 3,7 & 3,4 & 3.0 & 3,37 \\
\hline Contacto con el sufrimiento ajeno & 2,4 & 2,3 & 2,6 & 2,43 \\
\hline Relaciones con los profesionales en las practicas & 2,1 & 2,9 & 2,5 & 2,50 \\
\hline Impotencia e incertidumbre ante situaciones determinadas & 3,1 & 3,5 & 2,9 & 3,17 \\
\hline No saber controlar las relaciones con el paciente & 2,6 & 1,7 & 2,5 & 2,27 \\
\hline Implicaciones emocionales con el enfermo & 2,2 & 2,1 & 2,5 & 2.27 \\
\hline Riesgo a sufrir contagios, daños o lesiones en la relación con el paciente & 2,7 & 3,6 & 2,7 & 3.00 \\
\hline Riesgo a dañar al paciente & 3,1 & 3,2 & 3.0 & 3,10 \\
\hline Relaciones con familiares de los pacientes & 1,8 & 1,4 & 2,2 & 1,80 \\
\hline Exceso-sobrecarga de trabajo & 1,7 & 2,6 & 2,4 & 2,23 \\
\hline Resultados de la evaluación de las prácticas & 2,8 & 3,4 & 2,8 & 3.00 \\
\hline
\end{tabular}

Tabla 4. Porcentajes de factores estresantes por curso.

\begin{tabular}{|c|c|c|c|c|c|c|c|c|c|c|c|c|}
\hline & \multicolumn{4}{|c|}{10} & \multicolumn{4}{|c|}{20} & \multicolumn{4}{|c|}{30} \\
\hline & NADA & ALGO & BASTANTE & MUCHO & NADA & ALGO & BASTANTE & MUCHO & NADA & ALGO & BASTANTE & MUCHO \\
\hline 1. Manejo de nuevas tecnologías & $20 \%$ & $20 \%$ & $10 \%$ & $50 \%$ & $30 \%$ & $30 \%$ & $40 \%$ & $0 \%$ & $50 \%$ & $30 \%$ & $10 \%$ & $10 \%$ \\
\hline $\begin{array}{l}\text { 2. Desconocimiento ante una situación } \\
\text { clínica }\end{array}$ & $0 \%$ & $0 \%$ & $30 \%$ & $70 \%$ & $0 \%$ & $0 \%$ & $60 \%$ & $40 \%$ & $0 \%$ & $30 \%$ & $40 \%$ & $30 \%$ \\
\hline 3. Contacto con el sufrimiento ajeno & $20 \%$ & $30 \%$ & $40 \%$ & $10 \%$ & $40 \%$ & $10 \%$ & $30 \%$ & $20 \%$ & $10 \%$ & $40 \%$ & $30 \%$ & $20 \%$ \\
\hline $\begin{array}{l}\text { 4. Relaciones con los profesionales en las } \\
\text { prácticas }\end{array}$ & $30 \%$ & $40 \%$ & $20 \%$ & $10 \%$ & $0 \%$ & $40 \%$ & $30 \%$ & $30 \%$ & $20 \%$ & $30 \%$ & $30 \%$ & $20 \%$ \\
\hline $\begin{array}{l}\text { 5. Impotencia e incertidumbre ante } \\
\text { situaciones determinadas }\end{array}$ & $10 \%$ & $10 \%$ & $40 \%$ & $40 \%$ & $0 \%$ & $10 \%$ & $30 \%$ & $60 \%$ & $0 \%$ & $30 \%$ & $50 \%$ & $20 \%$ \\
\hline $\begin{array}{l}\text { 6. No saber controlar las relaciones con el } \\
\text { paciente }\end{array}$ & $20 \%$ & $20 \%$ & $40 \%$ & $20 \%$ & $50 \%$ & $30 \%$ & $20 \%$ & $0 \%$ & $0 \%$ & $60 \%$ & $30 \%$ & $10 \%$ \\
\hline $\begin{array}{l}\text { 7. Implicaciones emocionales con el } \\
\text { enfermo }\end{array}$ & $20 \%$ & $40 \%$ & $40 \%$ & $0 \%$ & $30 \%$ & $40 \%$ & $20 \%$ & $10 \%$ & $20 \%$ & $30 \%$ & $30 \%$ & $20 \%$ \\
\hline $\begin{array}{l}\text { 8. Riesgo a sufrir contagios, daños, o } \\
\text { lesiones }\end{array}$ & $30 \%$ & $10 \%$ & $20 \%$ & $40 \%$ & $0 \%$ & $0 \%$ & $40 \%$ & $60 \%$ & $0 \%$ & $60 \%$ & $10 \%$ & $30 \%$ \\
\hline 9. Riesgo a dañar al paciente & $20 \%$ & $10 \%$ & $10 \%$ & $60 \%$ & $0 \%$ & $20 \%$ & $40 \%$ & $40 \%$ & $10 \%$ & $0 \%$ & $80 \%$ & $10 \%$ \\
\hline $\begin{array}{l}\text { 10. Relaciones con los familiares de los } \\
\text { pacientes }\end{array}$ & $50 \%$ & $20 \%$ & $30 \%$ & $0 \%$ & $60 \%$ & $40 \%$ & $0 \%$ & $0 \%$ & $20 \%$ & $40 \%$ & $40 \%$ & $0 \%$ \\
\hline 11. Exceso - sobrecarga de trabajo & $50 \%$ & $30 \%$ & $20 \%$ & $0 \%$ & $20 \%$ & $30 \%$ & $20 \%$ & $30 \%$ & $20 \%$ & $30 \%$ & $40 \%$ & $10 \%$ \\
\hline $\begin{array}{l}\text { 12. Resultados de la evaluación de las } \\
\text { prácticas }\end{array}$ & $20 \%$ & $10 \%$ & $40 \%$ & $30 \%$ & $0 \%$ & $10 \%$ & $40 \%$ & $50 \%$ & $20 \%$ & $20 \%$ & $20 \%$ & $40 \%$ \\
\hline
\end{tabular}


Tabla 5.- Porcentaje de factores estresantes por sexo.

\begin{tabular}{|c|c|c|c|c|c|c|c|c|}
\hline & \multicolumn{4}{|c|}{ HOMBRE } & \multicolumn{4}{|c|}{ MUJER } \\
\hline & NADA & ALGO & BASTANTE & MUCHO & NADA & ALGO & BASTANTE & MUCHO \\
\hline Manejo de nuevas tecnologías & $42,85 \%$ & $42,85 \%$ & $0,00 \%$ & $14,28 \%$ & $30,43 \%$ & $21,74 \%$ & $26,09 \%$ & $21,74 \%$ \\
\hline Desconocimiento ante una situación clínica & $0 \%$ & $14,28 \%$ & $57,14 \%$ & $28,57 \%$ & $0,00 \%$ & $8,69 \%$ & $39,13 \%$ & $52,17 \%$ \\
\hline Contacto con el sufrimiento ajeno & $42,85 \%$ & $28,57 \%$ & $14,28 \%$ & $14,28 \%$ & $17,39 \%$ & $26,09 \%$ & $39,13 \%$ & $17,39 \%$ \\
\hline Relaciones con los profesionales en las prácticas & $14,28 \%$ & $42,85 \%$ & $28,57 \%$ & $14,28 \%$ & $17,39 \%$ & $30,43 \%$ & $30,43 \%$ & $21,74 \%$ \\
\hline Impotencia e incertidumbre ante situaciones determinadas & $14,28 \%$ & $14,28 \%$ & $57,14 \%$ & $14,28 \%$ & $0,00 \%$ & $17,39 \%$ & $34,78 \%$ & $47,83 \%$ \\
\hline No saber controlar las relaciones con el paciente & $57,14 \%$ & $42,85 \%$ & $0,00 \%$ & $0,00 \%$ & $8,69 \%$ & $34,79 \%$ & $39,13 \%$ & $17,39 \%$ \\
\hline Implicaciones emocionales con el enfermo & $42,85 \%$ & $42,85 \%$ & $14,28 \%$ & $0,00 \%$ & $17,39 \%$ & $34,79 \%$ & $34,79 \%$ & $13,04 \%$ \\
\hline $\begin{array}{l}\text { Riesgo a sufrir contagios, daños o lesiones en la relación con el } \\
\text { paciente }\end{array}$ & $14,28 \%$ & $28,57 \%$ & $42,85 \%$ & $14,28 \%$ & $8,69 \%$ & $21,74 \%$ & $17,39 \%$ & $52,17 \%$ \\
\hline Riesgo a dañar al paciente & $14,28 \%$ & $28,57 \%$ & $42,85 \%$ & $14,28 \%$ & $8,69 \%$ & $4,35 \%$ & $43,48 \%$ & $43,48 \%$ \\
\hline Relaciones con familiares de los pacientes & $71,42 \%$ & $28,57 \%$ & $0,00 \%$ & $0,00 \%$ & $34,79 \%$ & $39,13 \%$ & $26,09 \%$ & $0,00 \%$ \\
\hline Exceso-sobrecarga de trabajo & $71,42 \%$ & $14,28 \%$ & $14,28 \%$ & $0,00 \%$ & $26,09 \%$ & $34,79 \%$ & $21,74 \%$ & $17,39 \%$ \\
\hline Resultados de la evaluación de las prácticas & $14,28 \%$ & $0,00 \%$ & $42,85 \%$ & $42,85 \%$ & $13,04 \%$ & $17,39 \%$ & $30,43 \%$ & $39,13 \%$ \\
\hline
\end{tabular}

\section{REFERENCIAS BIBLIOGRÁFICAS}

[1] Ferrer Pascual, Ma A. Análisis situacional de las prácticas clínicas en la escuela de Enfermería de Soria. Metas, 2002;44:18-22.

[2] Zapico F. Aprendizaje y satisfacción de los estudiantes de enfermería en las prácticas clínicas. Hospital. Enfermería Clínica, 1997; 7:16-24.

[3] Juanola MD. Aprendizaje y satisfacción de los estudiantes de enfermería en las prácticas clínicas. Atención Primaria de Salud. Enfermería Clínica,1998; 8:254-62.

[4] Alemán S. Las prácticas de enfermería ¿crecimiento o deterioro? Situación de los alumnos previa a las prácticas. Enferm Cient. 1998.

[5] Gutiérrez MI. Las prácticas clínicas de enfermería: perspectiva de los estudiantes de primer curso. Metas de Enfermería. 2002; 49:50-3.

[6] López Medina IM. Percepción del estrés en estudiantes de enfermería en las prácticas clínicas. Enfermería Clínica, 2005; 15(6):307-13.

[7] Rodriguez A. Situaciones de las prácticas clínicas que provocan estrés en los estudiantes de enfermería. Enfermería Global, 2007.

[8] Zupiria Gorostidi X. Fuentes de estrés en la práctica clínica de los estudiantes de enfermería: evolución a lo largo de la diplomatura. Enfermería Clínica, 2006; 16(5):231-37. 